\title{
Arthroscopic staple fixation in the management of displaced anterior cruciate ligament avulsion fractures
}

\author{
Clevio Desouza ${ }^{1}$, Vinod Nair ${ }^{2, *}$, Amit Chaudhary ${ }^{3}$, Nitin Wadhwa ${ }^{4}$, Subhanshu Gupta ${ }^{5}$ \\ 1,4,5 Junior Resident, ${ }^{2}$ Associate Professor, ${ }^{3}$ Senior Resident, Dept. of Orthop aedics, Dr. D.Y. Patil Hospital, Pimpri, Pune, \\ Maharashtra, India
}

*Corresponding Author:

Email: nair.vinod904@gmail.com

\begin{abstract}
Introduction: Knee injuries are commonly associated with anterior cruciate ligament (ACL) avulsion fractures and its management is controversial ranging from conservative treatment to arthroscopic fixation. The purpose of our study was to ass ess the clinical and radiological results of arthroscopic staple fixation in the management of ACL avulsion fractures.

Materials and Methods: This study included twenty-two patients (17 males and 5 females) who were analyzed after undergoing arthroscopic staple fixation for displaced ACL avulsion fractures. The mean age was 32.2 years (15-55 years. The mean follow up was of 21 months (6-36 months). The patients were assessed clinically by calculating their Ly sholm and International Knee Documentation Committee (IKDC) scores. Radiological union was assessed in the follow up radiographs.

Results: The mean Lysholm score was 95.4 (83-100) and the mean IKDC score was 91.1 (77-100) at the final follow up. Anterior drawer's test was negative in 20 patients at the end of final follow up while two patients had grade I laxity. Seven cases had associated knee injuries. The final outcome was not greatly influenced by the presence of associated injuries when treated simultaneously. It was found that all the patients when examined at their final follow up were able to return to their pre-injury occupation.

Conclusion: The procedure of arthroscopic staple fixation in the management of displaced ACL avulsion fractures was found to be safe and reliable method for producing clinical and radiological outcome.
\end{abstract}

Keywords: ACL avulsion, Arthroscopy, Staple.

\section{Introduction}

Most of the injuries around the knee joint are contributed by anterior cruciate ligament (ACL) avulsion fractures and these result in significant disability if they are left untreated. These injuries may be caused by a direct blow over distal end of femur with the knee flexed or by forceful hyperextension of the knee. Previously, the management given to such patients included non-operative method of treat ment in the form of immobilization of the knee in full extension or in $20^{\circ}$ of flexion ${ }^{1}$ the position in which ACL is most relaxed. However, patients were immobilized for 4 to 6 weeks. Reduction is not achieved by manipulating the knee into hyperextension with the patient anesthetized, which can neither dislodge nor approximate the frag ment to its bed ${ }^{1}$ since the fractured frag ment is not between the articulating surface of tibia and femur, but lies in an empty non-articulating area of the jo int. There is a risk of knee stiffness and infection when tibial spine fractures are treated by open reduction and internal fixation. $^{2}$ These complications of long term immobilization are overcome by Arthroscopic fixation of ACL.

The purpose of our study was to assess the clinical and radiological results of arthroscopic staple fixation in the management of ACL avulsion fractures.

\section{Materials and Methods}

This study included Twenty- two patients (17 males and 5 females) who were analyzed after undergoing arthroscopic staple fixation for displaced
ACL avulsion fractures from January 2016 to January 2018 and had completed the minimum follow-up of six months and were available for final assessment. The mean age of the patients was 32.2 years (range 1555years). The mode of injury in these patients included road traffic accident in 19 patients and secondary to twisting injury in 3 patients.

All patients were assessed in the casualty by a careful physical examination to rule out associated injuries and by a plain radiograph of the knee jo int [Fig. 1]. On assessment, it was found that around thirteen patients had type II injury of the ACL (anterior third displacement of the avulsed frag ment) and nine patients had type III injury of the ACL where the avulsed fragment was displaced completely. There was exclusion of patients with polytrauma and contra lateral limb injuries from this study while patients with as sociated ipsilateral knee injuries were included.

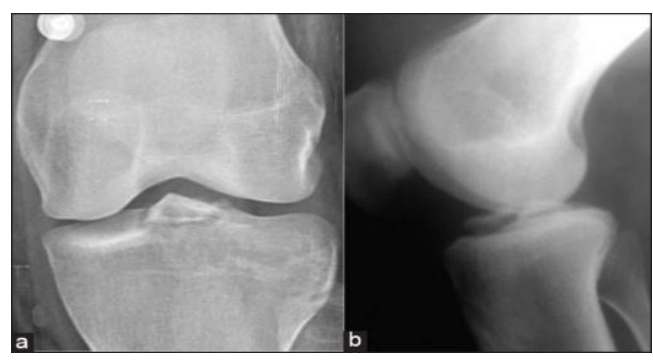

Fig. 1: Plain radiograph anteroposterior (a) and lateral view (b) of right knee of a 28 year old man with ACL avulsion fracture 
MRI study of four patients with severe communition of the avulsed fragment was done to assess the size of the largest bony fragment attached to the ACL that might allow fixation with a staple and also to assess the integrity of the ACL fibres. Seven patients were found to have as sociated ipsilateral knee injuries.

All patients underwent arthroscopic staple fixation. Tourniquet was used, after which control knee joint examination was done by standard medial and lateral para patellar arthroscopic portals. The medial portal was higher than the lateral portal and in a more vertical position to facilitate the staple fixation [Fig. 2]. Hematoma if present was drained by giving a thorough wash to the knee and the avulsed ACL fragment was visualized [Fig. 2b] and it was seen that there was no men iscal interposition. The titanium staple [Fig. 2c] is of 2 centimeters length and has two " $v$ "-shaped fangs. It was introduced through the high medial portal after threading it to the introducer and placed over the avulsed fragment. The staple was used as a joystick to reduce the fragment to its insertion bed athroscopically [Fig. 2d]. This was followed by the staple being driven through the avulsed fragment and into the tibial plateau by hammering on to the introducer gently. This was followed by unscrewing of the introducer from the staple head. Depending on the size of the fragment, the avulsed fragment was fixed with either one or two staples [Fig. 3]. In comminuted fractures, the largest fragment containing most of the ACL fibers was fixed with the staple and the remain ing loose small frag ments were removed, as it might lead to locking which may result in a fixed flexion deformity later. The stability of the fixation was checked intra operatively by pulling the ACL with a probe followed by performing of full range of motion of the knee.

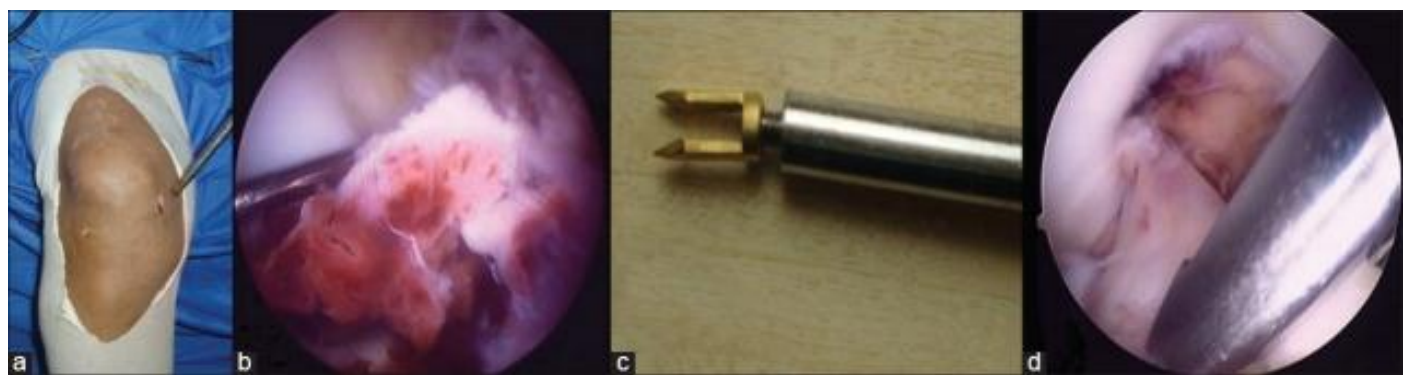

Fig. 2: (a) Arthroscopic high medial portal used to fix ACL avulsion fracture. (b) Arthroscopic view of ACL avulsion fracture. (c) Titanium staple used to fix ACL avulsion fracture. (d) Staple aiding in reduction of the avulsed fragment

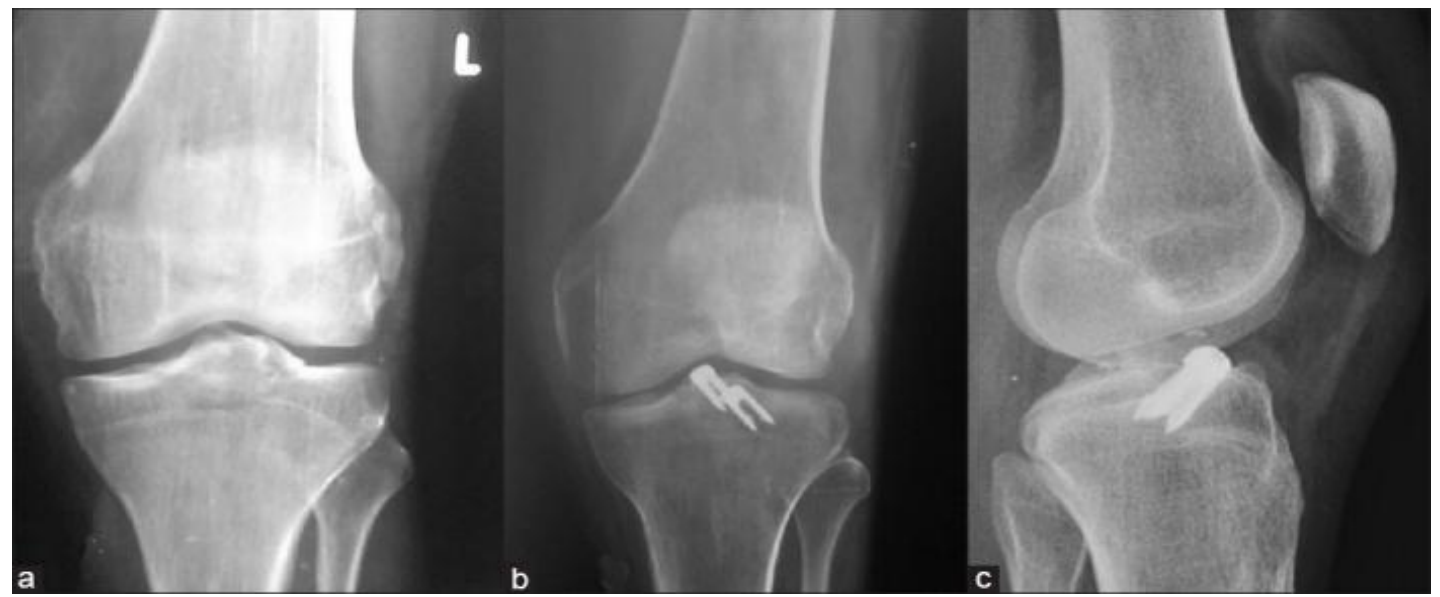

Fig. 3: (a) Anteroposterior X-ray of left knee of a 39 years old male shows large ACL avulsion, (b) Postoperative Anteroposterior and (c) lateral view of the same patient shows ACL fixed with 2 staples

The associated ipsilateral injuries of the knee were managed simultaneously. In our series as sociated knee injuries was found in seven patients. Out of these, three patients had tibial condyle fractures which were fixed percutaneously with screws before ACL avulsion fracture fixation for better purchase of the staple [Fig. 4]. Partial menisectomy was done in two patients who had meniscal injuries while two patients who had associated PCL avulsion fractures were fixed with cancellous screws by a posterior approach [Fig. 5]. 


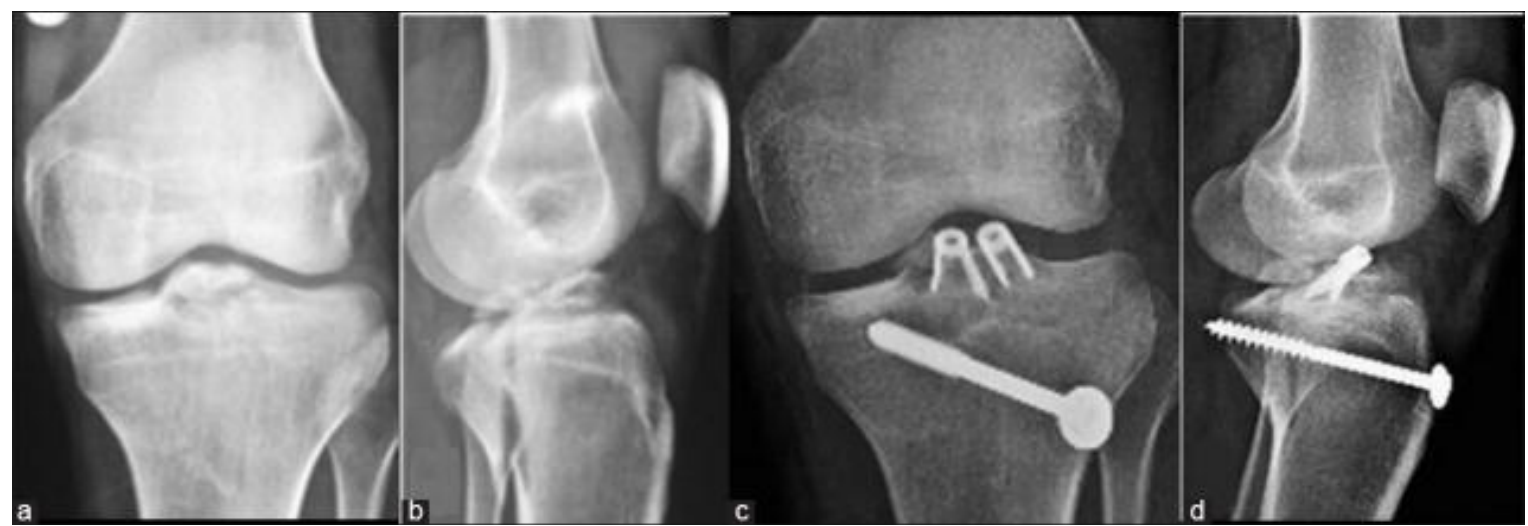

Fig. 4: Anteroposterior (a) and lateral (b) radiograph of the right knee of a 35 year old female shows ACL avulsion and posterior condyle fracture fixed respectively with staples (c,d) and percutaneous cancellous screws

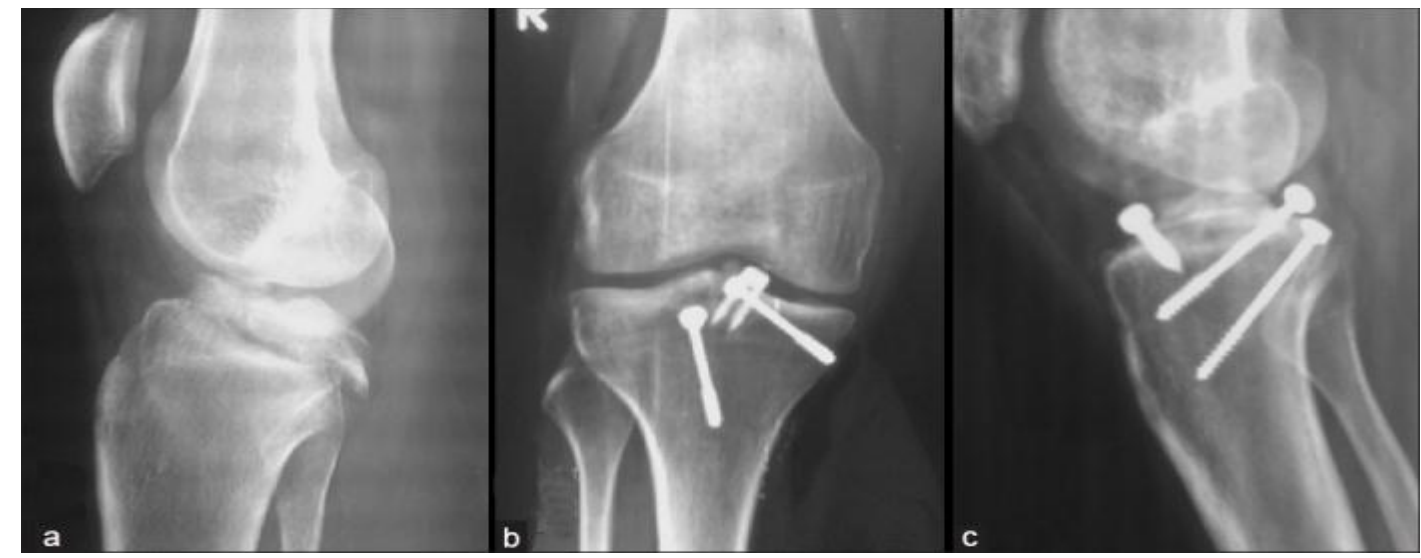

Fig. 5: ACL and PCL avulsion fracture of the right knee in a 47 year old male fixed with screws for PCL and staples for ACL

All patients were mobilized partial weight bearing for the first 4 weeks with a knee brace followed by full weight bearing along with knee bending exercises from 4 weeks postoperatively. This was followed by an active rehabilitation program which was started to gradually attain the full range of movements. Functional evaluation of the patients was done by examining for clinical signs of laxity and as sessment by the Lysholm score and the International Knee Documentation Committee (IKDC) score at end of third and sixth month and at final follow up. Follow up radiographs were done to assess the radiological healing in these patients.

\section{Results}

Twenty two patients were analyzed post operatively and followed up for a mean period of 21 months (range 6-36 months). Out of these patients, 20 patients were found to have complete range of motion of the knee joint at final follow up. Fixed flexion deformity of $5^{\circ}$ and restricted $15^{\circ}$ terminal flexion was seen in two patients. At the end of final follow up, anterior drawer's test was negative in 20 patients, while
2 patients revealed grade I laxity. Symptoms such as giving away of the knee on daily routine activities was not found in any of the patients and all of them returned to their pre-injury activity level. There were no surgical site infections in our series. The mean IKDC score was 91.1 (range 77-100) and the mean Lysholm score was 95.4 (range 83-100). The mean time to union was 8 weeks (range 6-10 weeks) radiologically and all cases united.

In our study, single staple fixation was done in 15 patients with s mall frag ment while 7 patients underwent double staple fixation for large avulsed fragment. The number of staples made no difference in the the functional outcome of the patients at final follow up. Patients with associated injuries returned back to their pre-injury occupation at time of final follow-up and the Lysholm and IKDC scores of these patients were comparable to those of the other patients with only ACL injuries [Table 1]. 
Table 1:

\begin{tabular}{|l|c|c|}
\hline \multicolumn{1}{|c|}{ Score } & Only ACL Injuries & With Associated Knee Injuries \\
\hline IKDC & 91.1 & 90.3 \\
\hline LYSHOLM & 95.4 & 94.4 \\
\hline
\end{tabular}

IKDC: International Knee Documentation Committee, ACL: Anterior cruciate ligament

One patient had back out of one staple 8 months after surgery in which two staples were used [Fig. 6]. The backed out staple was removed arthroscopically and the patient was able to return to his pre-injury activity level. Routine removal of the staple is not advocated. However we recommend removal of the staple if there is loosening or backing out radiologically. Otherwise the backed out staple can cause impingement and articular cartilage damage.

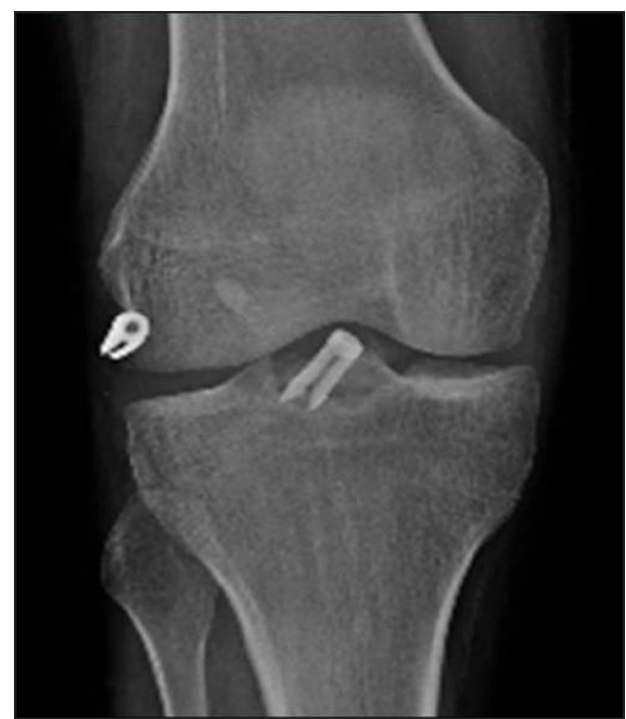

Fig. 6: Anteroposterior X-ray of the right knee showing back out of the staple in a 26 year old male

\section{Discussion}

The ACL is attached on the tibia to a wide depressed area in front of and lateral to the anterior tibial spine. When compared to PCL avulsions, ACL avulsion fractures account for a majority of knee injuries. ${ }^{3}$ Meyers and McKeever ${ }^{1}$ classified Avulsion fractures into three types as Type I-undisplaced, Type II - anterior third displacement, and Type III - completely displaced. Type I injuries are best treated conservatively.

Multiple methods have been described in the treatment of displaced fractured, these include cancellous screws, staples, sutures, $\mathrm{K}$ wires or bio absorbable suture anchors which are used in the fixation for ACL avulsions. Conservative management may be associated with complications such as prolonged immobilization, knee stiffness and residual instability. Variable degree of knee stiffness due to extensive dissection is seen in open methods of fixation which may require long period of immobilization. These drawbacks were overcome by Arthroscopic staple fixations.

ACL avulsions were more common in the age group of 15-30 years $(n=12)$ in our series. Kendall et al found the incidence of injury in adults higher than that in children ${ }^{4}$. Song EKet al found that there is no significant difference between adults and children in terms of final range of motion in avulsion fractures that were treated surgically. ${ }^{5}$ Wilfinger et $a l^{6}$ showed a good outcome at 1 year follow up in his study of 38 pediatric cases managed conservatively, however, there are no studies regarding conservative management in adults.

Huang et $a l^{7}$ reported good results with high functional scores with arthroscopic suture fixation. However as compared to staple fixation it is a difficult procedure. Patients treated by arthroscopic reduction and percutaneous pin fixation of ACL avulsions had side effects such as lack of extension, persistence of ligamentous instability, and quadriceps wasting when compared to arthroscopic staple fixation, this was shown in his study by Mc Lennan ${ }^{8}$. Excellent union rate for both acute and chronic cases of displaced tibial spine ACL avulsion fractures when treated by arthroscopic reduction with modified pull out suturing technique was shown by Ahn et al ${ }^{9}$. In our series, five patients were operated after 72 hours and they had slightly lower scores when compared to others. However, it does not affect the final outcome.

In our study the mean IKDC score was 91.1(77 to 100) and the mean Lysholm score was 95.4 (83 to 100) which is comparable to other series in the literature [Tables 2 and Table 3]. The mean IKDC score in the seven patients with associated injuries was 90.3 which was comparable to the total series mean score of 91.1 and a mean Lysholm score of 94.4 which was comparable to the total series mean score of 95.4 [Table 1] predicting that presence of associated knee injuries when treated simultaneously does not affect the final outcome of the ACL surgery.

Table 2: IKDC score

\begin{tabular}{|c|c|c|}
\hline IKDC Score & No of Cases & Percentage (\%) \\
\hline $91-100$ & 14 & 63.5 \\
\hline $81-90$ & 3 & 13.8 \\
\hline $71-80$ & 5 & 22.7 \\
\hline Total & 22 & 100 \\
\hline
\end{tabular}

IKDC: International Knee Documentation Committee 
Table 3: Lysholm score

\begin{tabular}{|l|c|c|}
\hline Lysholm score & No of Cases & $\begin{array}{c}\text { Percentage } \\
(\boldsymbol{\%})\end{array}$ \\
\hline $100-90$ & 14 & 63.6 \\
\hline $85-89$ & 6 & 27.3 \\
\hline $80-84$ & 2 & 9.1 \\
\hline Total & 22 & 100 \\
\hline
\end{tabular}

The number of staples used in the procedure to fix the fragment has no difference whatsoever on the functional outcome of the patients at final follow up. Song $e t a l^{5}$ using sutures and anchors for ACL avulsion obtained a Lysholm score of 89.5. Ahn et al produced a result of 95.6 Lysholm score using suture fixation. Robert $e$ t al published a Lysholm score of 94.2 using screws and suture alone. A satisfactory Lysholm score of 95.4 was obtained in our series with arthroscopic staple fixation. Seon et $a l^{10}$ compared screw and suture fixation and reported a Lysholm score of 91.7 and 92.7, respectively. In Medler series, avulsion fractures were fixed using ACL tibial drill guide and arthroscopy and have had good results. ${ }^{11}$ Good results were reported with bio absorbable suture anchors by Kim et al ${ }^{12}$ when compared in five cases.

When compared to open techniques of fixation, arthroscopic staple fixation facilitates earlier knee mobilization decreasing the chances of knee stiffness. The surgical time and length of hospitalization is also reduced in this procedure. Arthroscopic staple fixation is a technically simple procedure when compared to the screw fixation or suturing techniques. The difficulties such as insertion of long screws or the need of drilling or tapping can be overcome by staple fixation. Surgical expertise is required in performing the suture techniques and these are difficult as compared to staple fixation which can be easily reproduced.

Communition of the avulsed fragment, interposition of men isci or the transverse inter-meniscal ligament between fracture fragments, and intrasubstance ACL tear co mbined with an avulsion fracture are some of the common problems encountered in this technique.

When there is a severe communition of the fragment, ACL fibers can be detached from the fragments and the staples might not have a good purchase and may result in early back out of the staple. In such special circumstances, ACL reconstruction at later date is the preferred treatment of choice. Also, in the cases where avulsion of ACL is accompanied with ACL mid-substance tear, ACL reconstruction should be planned. When avulsion fracture is combined with a partial tear of ACL, the largest fragment can be fixed by a staple and the torn fibers can be debrided preventing the need for ACL reconstruction. This has been shown in where the two large avulsed fragments were fixed with staples and the remain ing intact fibers of ACL were preserved. These problems are circumvented by a preoperative MRI. So based on the study results we are now routinely taking preoperative MRI in all cases of ACL avulsions to rule out other ligament injuries, meniscal damage, and to document the integrity of the ACL fibers.

All patients obtained radiological union in a mean period of 8 weeks and all patients returned to their preinjury activity level at the final follow up. Based on our study, arthroscopic fixation is a preferred method to treat displaced ACL avulsion fractures when compared to the previous methods of open fixation in terms of earlier mobilization, avoiding knee stiffness and residual instability. Among the various arthroscopic techniques, staple fixation is a simple and effective procedure that when applied aptly gives good clinical and functional outcome.

\section{References}

1. Meyers MH, McKeever FM. Fracture of the Intercondylar Eminence of the tibia. J Bone Joint Surg Am. 1959;41:209-22.

2. Rademakers MV, Kerkhoffs GM, Kager J, Goslings JC, Marti RK, Raaymakers EL. Tibial spine fractures- a longterm followup study of open reduction and internal fixation. J Orthop Trauma. 2009;23:203-7.

3. Zaricznyj B. Avulsion fracture of the tibial eminence: Treatment by open reduction and pinning. J Bone Joint Surg Am. 1977;59:1111-4.

4. Kendall NS, Hsu SY, Chan KM. Fracture of the tibial spine in adults and children-A review of 31 cases. J Bone Joint Surg Br. 1992;74:848-52.

5. Song EK, Seon JK, Park SJ, Yoon TR. Clinical outcome of avulsion fracture of the anterior cruciate ligament between children and adults. J Pediatr Orthop B. 2009;18:335-8.

6. Wilfinger C, Castellani C, Raith J, Pilhatsch A, Höllwarth ME, Weinberg AM. Nonoperative treatment of tibial spine fractures in children-38 patients with a minimum follow-up of 1 year. J Orthop Trauma. 2009;23:519-24.

7. Huang TW, Hsu KY, Cheng CY, Chen LH, Wang CJ, Chan YS, et al. Arthroscopic suture fixation of tibial eminence avulsion fractures (36 cases) Arthroscopy. 2008;24:1232-8.

8. McLennan JG. The role of arthroscopic surgery in the treatment of fractures of the intercondylar eminence of the tibia. J Bone Joint Surg Br. 1982;64:477-80.

9. Ahn JH, Yoo JC. Clinical outcome of arthroscopic reduction and suture for displaced acute and chronic tibial spine fractures. Knee Surg Sports Traumatol Arthrosc. 2005;13:116-21.

10. Seon JK, Park SJ, Lee KB, Gadikota HR, Kozanek M, Oh LS, et al. A clinical comparison of screw and suture fixation of anterior cruciate ligament tibial avulsion fractures. Am J Sports Med. 2009;37:2334-9.

11. Medler RG, Jansson KA. Arthroscopic treatment of fractures of the tibial spine. Arthroscopy. 1994;10:292-5.

12. In Y, Kim JM, Woo YK, Choi NY, Moon CW, Kim MW. Arthroscopic fixation of ACL avulsion fractures using bio absorbable suture anchors. Knee Surg Sports Traumatol Arthrosc. 2008;16:286-9. 